\title{
Temperature Dependence of Light Absorption by Water
}

\author{
J.B.Cumming \\ Chemistry Department, Brookhaven National Laboratory, Upton, New York 11973
}

(Dated: June 30, 2021)

\begin{abstract}
A model is described that relates the temperature coefficient of the optical absorption spectrum of pure water to the frequency derivative of that spectrum and two parameters that quantify the dependence of a peak's amplitude and its position on temperature. When applied to experimental temperature coefficients, it provides a better understanding of the process than the analysis currently in use.

Keywords: water, optical absorption, temperature coefficient
\end{abstract}

\section{INTRODUCTION}

Understanding the temperature dependence of optical absorption takes on increasing importance as more accurate spectra become available. A high quality absorption spectrum from the infrared to the blue for water at $22^{\circ} \mathrm{C}$ can be obtained by combining the data of Kou, Labrie, and Chylek [1] with that of Pope and Fry [2]. Kou et al. studied the range from 666 to $2400 \mathrm{~nm}$ with a Fourier-transform infrared spectrometer. They derived absorption coefficients from the ratio of transmittances obtained with different path lengths. This exactly cancels reflection losses at the air-window and window-water interfaces and absorption by the cell windows. Only transmittance values between $20 \%$ and $60 \%$ were used to avoid saturation and base line uncertainties. Absorption coefficients from 380 to $727.5 \mathrm{~nm}$ were obtained by Pope and Fry using a novel integrating cavity absorption meter(ICAM). In the ICAM, optical power loss from a water-filled highly-reflective cavity is measured as a function of wavelength. Scattering, which is important at the lower end of this wavelength range, does not cause a loss of power, hence the ICAM measures absorption.

The absorption coefficient of water decreases by some six decades from the infrared through the blue. The infrared spectra in Fig. 1 from Kou et al. [1] show a general exponential decrease vs frequency that is modulated by various peaks, valleys, and shoulders indicating absorption by discrete states or groups of states. The amplitude of these features decreases relative to the background continuum with increasing frequency. The use of frequency (in wavenumbers) emphasizes the approximately uniform spacing of these features.

The vibrational level spectrum of an isolated water molecule can be described in terms of harmonics or combinations of three normal modes: a symmetric and an asymmetric stretch, and a bending or scissors mode. Single levels are broadened by rotational structure. In condensed phases, formation and breaking of hydrogen bonds further broadens levels and may introduce additional modes. The result is not a continuum for low exci-

*Electronic address: cumming@bnl.gov

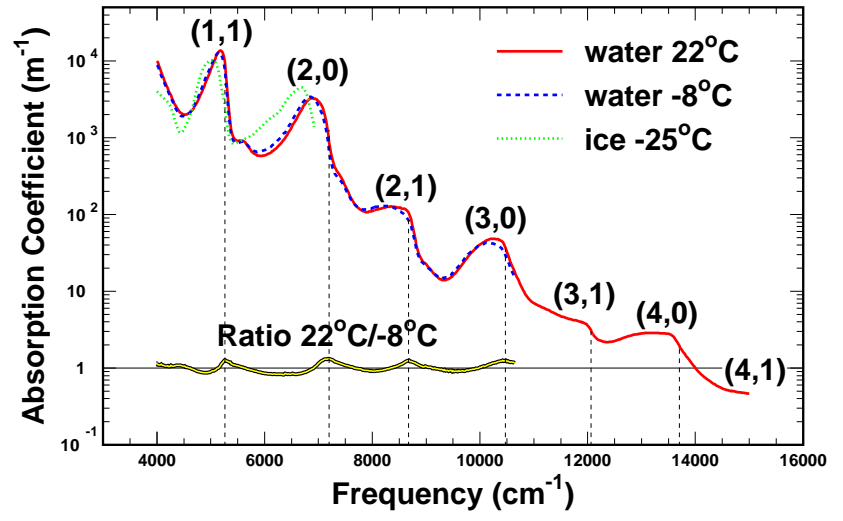

FIG. 1: Absorption spectra of water, supercooled water, and ice from Kou, Labrie, and Chylek [1]. Oscillator assignments are shown in parentheses above the peaks, see text. The ratio of the $22^{\circ}$ spectrum to the $-8^{\circ}$ spectrum is shown in the lower part of the figure. Vertical dashed lines mark inflection points on the high frequency side of each $22^{\circ}$ peak.

tations: Spectra in Fig. 1 retain various discrete features. Patel and Tam [3] have identified absorption peaks in the liquid with the band structure of two anharmonic oscillators. The first was based on a single (degenerate) stretching mode. The second, a parallel series of states, was upshifted by a bending excitation. We will refer to these as the stretching band and the combination band, respectively. The frequency in wavenumbers of levels in terms of stretching and bending numbers, $\left(n_{s}, n_{b}\right)$, is given by:

$$
\begin{aligned}
& \nu\left(n_{s}, 0\right)=n_{s}\left(3620-63 n_{s}\right) \\
& \nu\left(n_{s}, 1\right)=\nu\left(n_{s}, 0\right)+1645 .
\end{aligned}
$$

The peaks and shoulders in Fig. 1 are labeled with their $\left(n_{s}, n_{b}\right)$ assignments.

The $22^{\circ} \mathrm{C}$ absorption spectrum of Pope and Fry [2] at has a minimum in the vicinity of $420 \mathrm{~nm}$. Recent data from Abe and the Super-Kamiokande Collaboration [4] at $13^{\circ} \mathrm{C}$, and from Griskevich, Renshaw, and Smy [5] at $15^{\circ} \mathrm{C}$, suggest that this minimum is appreciably deeper and shifted to a higher frequency. The present paper is an outgrowth of a study of scattering and absorption by water in the vicinity of the minimum. When comparing various measurements, it was desirable to separate 
the influence of temperature from those due to other systematic effects. The classical treatment of temperature dependence is to fit the absorption spectrum to an appropriate set of Gaussians and then to determine a unique temperature coefficient for each peak from experimental data. An example of this sort of analysis due to Jonasz and Fournier [6] is described in Sec. 3. Measurements of Buiteveld, Hakvoort, and Donze [7], of Pegau, Gray, and Zaneveld [8], and of Langford, McKinley, and Quickenden [9] indicate that absorption peaks in the visible not only shift upward, but also move to higher frequencies with increasing temperature. The model developed below predicts that shifts of peak position may result in apparent positive and negative temperature coefficients for a given peak. A variety of experimental observations are a natural consequence of this model.

\section{THE MODEL}

Ratios of absorption coefficients at $22^{\circ} \mathrm{C}$ to those at $-8^{\circ} \mathrm{C}$ are plotted in the lower part of Fig. 1. Although the individual absorption spectra show considerable structure, the ratio takes a simple form; a series of cusps where the ratio is greater than one, separated by regions where it drops below unity. Vertical dashed lines in Fig. 1 indicate that the cusps correspond with inflection points on the high frequency sides of peaks in the $22^{\circ} \mathrm{C}$ spectrum, not their centers. This suggested a connection between temperature dependence and the shape of the absorption spectrum.

Consider part of an absorption spectrum, $a(\nu, T)$, in the vicinity of some spectral feature located at frequency $\nu_{0}$ where the absorption coefficient is $a_{0}$. We assume that $a$ is separable:

$$
a(\nu, T)=a_{0}(T) f\left(\nu-\nu_{0}(T)\right) .
$$

Differentiating Eq. 2 with respect to $T$ and substituting $d f / d \nu_{0}=-d f / d \nu$ leads to:

$$
\frac{1}{a} \frac{d a}{d T}=\frac{1}{a_{0}} \frac{d a_{0}}{d T}-\frac{1}{a} \frac{d a}{d \nu} \frac{d \nu_{0}}{d T} .
$$

The temperature coefficient ${ }^{1}$ is the sum of two terms. The first is the trivial "classical" interpretation: If a temperature change of $+1^{\circ} \mathrm{C}$ increases the intensity of an absorbing peak by $+1 \%$, then the temperature coefficient is $+1 \%$ per ${ }^{\circ} \mathrm{C}$ across the whole peak.

The second term on the right side of Eq. 3 has more interesting properties. If the region of interest contains a peak, $d a / d \nu$ is zero at the peak position. The temperature coefficient will change sign in scanning across that

\footnotetext{
${ }^{1}$ We will use "temperature coefficient" for the fractional temperature coefficient, reserving "absolute temperature coefficient" for $d a / d T$.
}

peak. That maximum temperature changes match the inflection points on the high frequency side of the peaks in Fig. 1, indicates that $d \nu_{0} / d T$ is positive in the infrared. Experimental data presented in Sec. 3 indicate this is true in the visible as well. As a consequence, temperature coefficients will be negative on the low frequency side of a peak and positive on the high side.

If the region of interest is a decreasing continuum, the derivative is negative throughout, and temperature coefficients will be positive. Consider then the progressive transition from a spectral region dominated by individual peaks to one of a decreasing continuum. In the first region, temperature coefficients should show a bipolar oscillation, with some upwards bias due to the underlying continuum and any contribution from the classical term of Eq.3. Moving up in frequency, the relative contribution of peaks decreases and that of the continuum increases. The amplitude of the oscillations will decrease, and the upward bias will increase. At some point, the negative lobes will be balanced out by the bias, and an absorption peak will appear to have only a positive temperature coefficient peak. However, this will not be centered at the peak position but on the inflection point on its high frequency side. With further increases in frequency, the envelopes of the upper and lower coefficient oscillations will converge to the continuum value.

\section{EXPERIMENTAL DATA}

The logarithmic frequency derivative, $(1 / a)(d a / d \nu)$, is compared with the fractional temperature coefficient, $(1 / a)(d a / d T)$, in Fig. 2. The derivative of the $22^{\circ} \mathrm{C}$ spectrum in Fig. 1 is shown as a hollow curve in Fig. 2(a). The solid curve is from the absorption spectrum of Pope and Fry [2], also at $22^{\circ} \mathrm{C}$. Peak positions of the stretching and combination band absorptions are shown by $\left(n_{s}, n_{b}\right)$ labels. Each of the oscillator states is marked by a sharp negative derivative spike on its high frequency side. Positive excursions are less pronounced due to, in part, the overall decreasing trend of the spectrum. These derivative oscillations damp down in moving into the visible. Negative excursions corresponding to each state persist, but the positive excursions transform into a small negative background between the larger negative excursions. Pronounced peaks and valleys have given way to an exponentially decreasing absorption spectrum where specific absorbing states appear only as shoulders. Note that the amplitude of negative derivative peaks associated with $\left(n_{s}, 1\right)$ transitions decreases more rapidly than those for $\left(n_{s}, 0\right)$. They are decidedly less pronounced in the visible. This suggests that the dependence on frequency is different for the two bands. Positions of the derivative minima are projected on to Fig. 2(b) by vertical dashed lines.

The dependence of the temperature coefficient on frequency is shown in Fig. 2(b). The hollow curve was obtained from the data of Kou et al.[1]. The solid curve 

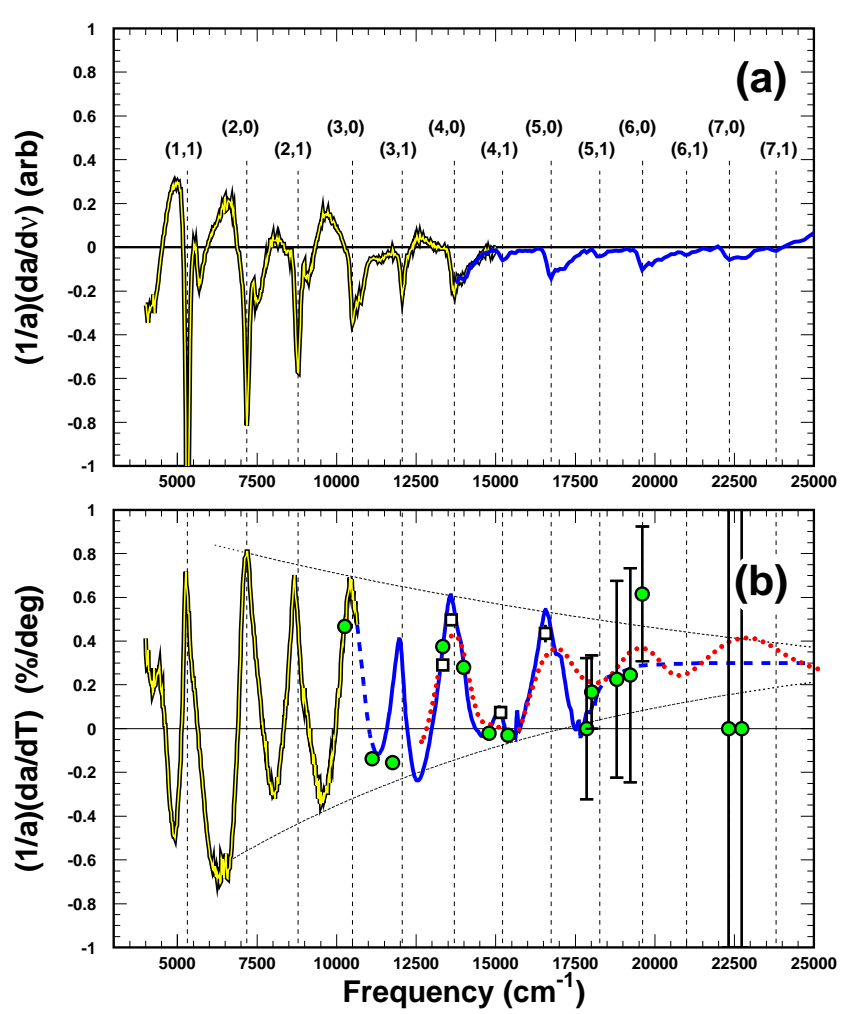

FIG. 2: Comparison of the frequency derivative of the water absorption spectrum at $22^{\circ} \mathrm{C}$ with the temperature coefficient of that spectrum. Hollow curves in (a) and (b) are from Kou et al. [1]. The solid curve in (a) is from Pope and Fry [2]. The solid curve in (b) is based on the measurements of Langford et al. 99]. A dashed curve connects it to the hollow curve. Circles show experimental values from Pegau et al. [8], squares those from Trabjerg and Hojerslev [10]. The heavy dotted curve is from an analysis by Jonasz and Fournier [6]. Thin dotted curves show the approximate envelopes of the temperature coefficient oscillations. Positions of frequency derivative minima are shown by vertical thin dashed lines.

is based on the extensive measurements of Langford, McKinley, and Quickenden [9]. These authors studied the temperature dependence of decadic absorptivity, $A$, over the wavelength range from 550 to $900 \mathrm{~nm}$ and for temperatures between 15 and $60^{\circ} \mathrm{C}$. At any wavelength, $A$ was observed to vary linearly with $T$. Their published decadic $d A / d T$ values were converted to $d a / d T$, then to $(1 / a)(d a / d T)$ using the absorption coefficients of Kou et al. and of Pope and Fry. A spline interpolation joining the two measurements is shown as a heavy dashed line in Fig. 2(b).

Buiteveld et al. [7] have derived $d a / d T$ values for wavelengths from 400 to $800 \mathrm{~nm}\left(12,500\right.$ to $\left.25,000 \mathrm{~cm}^{-1}\right)$ from absorption spectra obtained between 2.5 and $45^{\circ} \mathrm{C}$. They noted that $d a / d T$ was nearly constant at $0.0012 \mathrm{~m}^{-1}$ per ${ }^{\circ} \mathrm{C}$ below $580 \mathrm{~nm}$. Pegau et al. have concluded there was a systematic bias of 0.0011 in that value. We sub- tracted that bias prior to converting the Buiteveld et al. $d a / d T$ values to $(1 / a)(d a / d T)$. These were in agreement those of Langford et al. for wavelengths longer than 580 $\mathrm{nm}$. They have not been included in Fig. 2(b) because of uncertainties due to the baseline correction,

Absolute temperature coefficients from Pegau et al. [8] were converted to the $(1 / a)(d a / d T)$ values which are shown as circles in Fig. 2(b). With the exception of the point at $850 \mathrm{~nm}\left(11,760 \mathrm{~cm}^{-1}\right)$, there is good agreement with the curve based on Kou et al. and Langford et al. Four points due to Trabjerg and Hojerslev [10], squares in Fig. 2(b), are also in agreement with that curve.

Jonasz and Fournier [6] have reported a detailed analysis of the absorption spectrum of water and its temperature coefficient in the range from 380 to $800 \mathrm{~nm}$ The absorption spectrum could not be reproduced with a ten-member basis-set of Gaussians corresponding to the stretching and combination band states $(4,0)$ to $(8,1)$. Five additional Gaussians were needed to obtain "impressive" agreement with the experimental spectrum. Jonasz and Fournier then used available data to derive fractional temperature coefficients for each of the 15 Gaussians. This classical approach is equivalent to setting $d \nu_{0} / d T=0$ in Eq.3. Their derived spectrum of temperature coefficients, the dotted curve in Fig. 2(b), is a reasonable fit to the experimental data for frequencies below $15,500 \mathrm{~cm}^{-1}$ where the extra Gaussians were added. In particular, it reproduces the negative values to the left of the $(4,0)$ peak and the near zero values in the vicinity of the $(4,1)$ position. Agreement is poorer at higher frequencies. Oscillations of their curve in that region are a consequence of their ascribing temperature coefficients of $0.45 \%$ per ${ }^{\circ} \mathrm{C}$ to the $\left(n_{s}, 0\right)$ absorptions and $0.20 \%$ per ${ }^{\circ} \mathrm{C}$ to the $\left(n_{s}, 1\right)$ absorptions in their original ten-member basis set. The oscillations would have continued down to $12,500 \mathrm{~cm}^{-1}(800 \mathrm{~nm})$ were it not for the five extra Gaussians. One positioned near the $(4,0)$ peak had a larger positive coefficient. The other four located between the $(4,0)$ and $(5,0)$ peaks had large negative values. The physical significance of unusual properties of these phantom states is not clear.

Several features of Fig. 2 support the derivative model. The one-to-one correspondence between frequency derivative minima and temperature coefficient maxima is striking and implies that $d \nu_{0} / d T$ in Eq. 3 is positive. The nearly symmetric, two-lobed form of the temperature coefficient spectrum in the infrared provides strong evidence that frequency shift is a major contributor to temperature dependence. The region of near zero temperature coefficients at $\sim 15,000 \mathrm{~cm}^{-1}$ is interpreted as being due to a balance between the negative lobe of the $(5,0)$ absorption and and the positive contribution from the decreasing continuum. The $(4,1)$ absorption makes a small contribution in this valley.

Approximate upper and lower envelopes of the temperature coefficient oscillations for the $\left(n_{s}, 0\right)$ absorptions are shown as thin dashed curves in Fig. 2(b). The damping of oscillations with increasing frequency, and the the 
approach to a limit of $\sim+0.3 \%$ per ${ }^{\circ} \mathrm{C}$ which is shown as a heavy dashed line, are consistent with the change from discrete absorption peaks in the infrared to a decreasing continuum in the visible. Note that the $0.3 \%$ per ${ }^{\circ} \mathrm{C}$ applies to absorption only. Because scattering has little if any temperature dependence, the fractional temperature coefficient for attenuation is reduced by the ratio of absorption to attenuation. For example, scattering and absorption coefficients are equal at $425 \mathrm{~nm}$ [4] and the fractional temperature coefficient for attenuation is then $0.15 \%$ per $^{\circ} \mathrm{C}$.

\section{CONCLUSIONS}

The temperature dependence of optical absorption by water has a complex dependence on frequency related to properties of the absorbing states or continuum. The present model, which connects the temperature dependence to the frequency derivative of the absorption spec- trum, provides a framework for understanding this dependence. The composite curve in Fig. 2(b) based on Kou et al. and Langford et al. with the dashed interpolation and extrapolation, provides practical temperature corrections for absorption coefficients. The model suggests that analyses of the type which fit an absorption spectrum with Gaussians should use the derivative of the Gaussians to fit the temperature coefficient spectrum. A continuum background must be included in both.

\section{Acknowledgments}

The author wishes to thank M. Smy for communicating his unpublished results. This work, conducted at Brookhaven National Laboratory, was supported by the U.S. Department of Energy's Offices of Nuclear Physics and High Energy Physics, under Contract DE-AC0298CH10886.
[1] L.Kou, D.Labrie, and P.Chylek, Appl. Opt. 32, 3531 (1993).

[2] R.M.Pope and E.S.Fry, Appl. Opt. 36, 8710 (1997).

[3] C.K.N.Patel and A.C.Tam, Nature 280, 302 (1979).

[4] K.Abe and the Super-Kamiokande Collaboration, Phys. Rev. D 83, 052010 (2011).

[5] J.Griskevich, A.Renshaw, and M.Smy (2012), private communication.

[6] M. Jonasz and G. Fournier, Light Scattering By Particles In Water: Theoretical and Experimental Founda- tions (Academic, 2007).

[7] H.Buiteveld, J.H.M.Hakvoort, and M.Donze, SPIE Ocean Optics XII 2258, 174 (1994).

[8] W.S.Pegau and D.Gray and J.R.Zaneveld, Appl. Opt. 36, 6035 (1997).

[9] V.S.Langford, A.J.McKinley, and T.I.Quickenden, J.Phys.Chem A 105, 8916 (2001).

[10] I.Trabjerg and N.K.Hojerslev, Appl. Opt 35, 2653 (1996). 